\title{
Genital and Extragenital Canine Transmissible Venereal Tumor in Dogs in Grenada, West Indies
}

\author{
Alfred Chikweto*, Sachin Kumthekar, Hugh Larkin, Claude Deallie, Keshaw P. Tiwari, \\ Ravindra N. Sharma, Muhammad I. Bhaiyat \\ Pathobiology Academic Program, School of Veterinary Medicine, St. George's University, St. George, Grenada \\ Email: *achikweto@sgu.edu
}

Received December 20, 2012; revised January 21, 2013; accepted February 21, 2013

Copyright (C) 2013 Alfred Chikweto et al. This is an open access article distributed under the Creative Commons Attribution License, which permits unrestricted use, distribution, and reproduction in any medium, provided the original work is properly cited.

\begin{abstract}
Transmissible venereal tumor (TVT) is a widely reported transplantable tumor in dogs, with no breed or sex predilection and with a low metastatic rate. This retrospective study conducted from 2005 and 2011 sought to evaluate the total number of TVT cases, and to report extragenital TVT lesions in dogs from the small tropical island of Grenada, West Indies. Evaluation of the biopsy and necropsy specimens from dogs with TVT was carried out by cytological and histopathological methods. In the present study, we observed a total of 78 dogs with TVT, comprising 40 male and 38 female dogs. Most cases were from dogs between 3 and 6 years; less frequently from older dogs (over 6 years) or younger dogs $(1-2$ years of age). In the present study, we report 15 cases $(19.2 \%)$ of dogs with TVT in extragenital sites. Tumors were noted in the nasal cavity, eye orbit, spleen, liver, skin, ribs, subcutaneous, and submandibular, cervical and inguinal lymph nodes. There was also evidence of metastasis to the ovaries in 2 dogs. The most interesting finding was the presence of extragenital lesions without primary genital involvement in 4 cases (5.1\%). Our findings emphasize the need to consider TVT on the list of differential diagnosis for masses in extragenital sites in dogs from geographic regions where TVT is prevalent.
\end{abstract}

Keywords: Dog; Extragenital; Transmissible Venereal Tumor; Grenada

\section{Introduction}

Transmissible venereal tumor (TVT) is a round cell tumor of probable histiocytic origin that affects dogs and other canids [1,2]. It is transmitted by inoculation of intact neoplastic cells into damaged mucosae or skin mainly through coitus and occasionally by social behavior such as sniffing and licking [3]. The tumor is mainly located on the external genitalia and is generally considered benign [4]. However, it has been occasionally reported in extragenital locations and internal genital organs $[3,5]$.

Geographically, TVT occurs predominantly in tropical and subtropical countries where many freely roaming dogs with unrestricted sexual activity are found [6]. Mature male and female dogs are equally affected and there is no breed predisposition to TVT [4]. Predisposing facors for the genital and extragenital occurrence of TVT can be due to the increased number of stray dogs; age; and immunological status, which could be a contributing

${ }^{*}$ Corresponding author. factor to the unusual metastasis to distant sites $[3,7,8]$. There is a paucity of TVT studies in the West Indies; apart from reports on the prevalence from Puerto Rico and from the Bahamas $[9,10]$. The main objectives of this study were to evaluate the total number of TVT cases and to report lesions located on extragenital sites.

\section{Materials and Methods}

Necropsy and biopsy records of dogs with masses suspected to be neoplastic processes and submitted to the pathology laboratory at the School of Veterinary Medicine from 2005 to 2011 were evaluated. History relating to sex including neuter status, breed and age was also noted. Samples were submitted from the Small Animal Hospital, the Grenada Society for Prevention of Cruelty to Animals and from private clinics. Tissues had been fixed in 10\% buffered formalin, processed, sectioned at 3 $\mu \mathrm{m}$, and stained with hematoxylin and eosin. All cases with a confirmed histopathologic and/or cytologic diagnosis of TVT were included in this study. Since TVT is transmitted mainly through coitus, all sexually immature 
dogs were excluded from the study.

A Chi-squared test of significance was used to compare differences in age groups of dogs affected with TVT.

\section{Results}

On cytology, TVT cells showed a predominance of round cells with abundant pale cytoplasm and distinct, punctate cytoplasmic vacuoles. On histopathology, neoplastic cells appeared as round cells often divided into packets by fine fibrous stroma. We diagnosed 78 dogs with TVT, comprising 40 males and 38 females. Among the 78 TVT cases there was evidence of lesions located in extragenital sites in 15 dogs. Extragenital lesions without primary genital involvement in 4 cases were also observed. Two female dogs with primary genital TVT lesions had distant metastasis to the ovaries. Characteristics and distribution of lesions in the affected dogs are as shown in Tables 1 and 2. Most cases were between 3 - 6yrs (41 dogs) followed by $1-2$ yrs (21 dogs) and $>6$ yrs (16 dogs) of age. At the time of presentation, it was observed that 30 females and 37 males were intact whereas 8 females and 3 males were neutered. The majority of cases ( $92 \%)$ were of the mixed local breed referred to as the Grenadian Pothound. Pot hounds are the most common type of dogs in Grenada. They are often owned but generally allowed to wander at will. Other breeds represented were German shepherd dog (2 dogs), Rottweiler (2 dogs), Golden retriever $(1 \mathrm{dog})$ and Pit bull terrier $(1 \mathrm{dog})$.

Table 1. Total number of tumor cases and percentage of cases of genital and extragenital TVT in dogs from Grenada, West Indies.

\begin{tabular}{ccccc}
\hline Total number of tumor cases & Total of TVT cases & Male & Female & Percentage of TVT cases \\
\hline 420 & 78 & 40 & 38 & $78 / 420(18 \%)$ \\
\hline TVT cases in with extragenital lesions & & Male & Female & Percentage of extragenital TVT \\
\hline 15 & & 8 & 7 & $15 / 78(19.2 \%)$ \\
\hline
\end{tabular}

Table 2. Cases of 15 dogs with TVT in extragenital sites from Grenada, West Indies.

\begin{tabular}{|c|c|c|c|c|c|}
\hline Case No. & Breed & Age (yrs) & Sex & Primary genital lesion & Secondary location \\
\hline 1 & Mixed & 3 & $\mathrm{~F}$ & Vagina & $\begin{array}{c}\text { Spleen, liver, } \\
\text { submandibular lymphnodes, } \\
\text { right eye, subcutaneous }\end{array}$ \\
\hline 2 & Mixed & 2 & $\mathrm{~F}$ & Vagina & Caudal mammary glands \\
\hline 3 & Mixed & 5 & $\mathrm{~F}$ & Vagina & Subcutaneous \\
\hline 4 & GSD & 2 & $\mathrm{~F}$ & Vagina & $\begin{array}{l}\text { Right ovary, skin, } \\
\text { subcutaneous }\end{array}$ \\
\hline 5 & Mixed & 3 & $\mathrm{~F}$ & None & Subcutaneous \\
\hline 6 & Pitbull & 6 & $\mathrm{~F}$ & Vagina & $\begin{array}{c}\text { Ovaries, caudal mammary glands, } \\
\text { subcutaneous }\end{array}$ \\
\hline 7 & Mixed & 1 & $\mathrm{~F}$ & Vagina & Right eye \\
\hline 8 & Mixed & 3 & M & Penis & Subcutaneous and ribs \\
\hline 9 & Mixed & 3 & M & None & $\begin{array}{l}\text { Liver, spleen, lungs, } \\
\text { nasal cavity, cervical lymph nodes }\end{array}$ \\
\hline 10 & Mixed & 12 & M & Penis & Subcutaneous, \\
\hline 11 & Mixed & 3 & M & Penis & $\begin{array}{l}\text { Subcutaneous, liver, } \\
\text { anterior mediastinum }\end{array}$ \\
\hline 12 & Mixed & 4 & M & None & Subcutaneous \\
\hline 13 & Mixed & 4 & M & None & Subcutaneous \\
\hline 14 & Mixed & 6 & M & Penis & $\begin{array}{l}\text { Subcutaneous, liver, } \\
\text { inguinal lymphnodes }\end{array}$ \\
\hline 15 & Mixed & 3 & M & Penis & Spleen \\
\hline
\end{tabular}

GSD $=$ German shepherd dog 


\section{Discussion}

Canine transmissible venereal tumor is most common in dogs 2 to 5 years old and there is no breed or sex predisposition [7]. This is quite similar to our study which revealed dogs in the age range of 3 to 6 years old as highly significantly affected compared to $1-2$ years and greater than 6 years $(\mathrm{P}<0.01$, chi square). Eleven dogs in this study were neutered, but it is likely that most of the dogs were intact at the time they developed TVT. Although TVT can affect any breed, a substantial percentage (92\%) of the cases in this study were Grenadian Pot hounds; thus making it difficult to evaluate breed predisposition.

In the present study, $16.7 \%$ of dogs diagnosed with TVT had extragenital lesions either by metastasis from a primary genital lesion or through implantation following a social behavior. Metastasis of TVT from primary genital sites is uncommon but is usually seen in the regional lymphnodes [4]. Other reported sites of metastasis include skin, subcutaneous, distant lymphnodes, brain, eyes, spleen, liver, testicles, musculature, lungs, kidneys, anus, bones, mammary glands $[6,8,11-14]$. In the current study, TVT was detected in most of the sites reported above apart from metastasis to the rectum, anus, kidneys and the brain. Metastasis of TVT to the subcutaneous was the most common in our study.

Reports of primary TVT arising in extragenital sites are few; the most common sites being the nasal and oral cavities, skin, and the rectum; transmitted by sniffing or licking [15]. In the current study, 1 dog had primary TVT arising from the nasal cavity with disseminated distant metastasis whereas 3 dogs had primary TVT in the subcutaneous tissue. Similary, Papazoglou et al. (2001) have reported primary TVT from the nasal cavity with distant metastasis in 6 dogs [16]. In a retrospective study of 29 cases Rogers et al. (1998) found 2 cases with primary extragenital TVT in the nasal cavity with metastasis to the cervical lymphnodes and subcutaneous tissue in 1 dog [4]. Recently, Gupta and Sood reported primary TVT of the mammary glands without evidence of TVT in the external genitalia in 2 dogs from India [17]. Although the external genitalia are the most commonly affected by TVT, metastasis is rarely seen in internal genital organs. To our knowledge there is only one published report on TVT involving the uterus and ovaries [5]. In this study we also report TVT metastatic to the ovaries in 2 dogs.

The biological behavior of TVT is quite variable and depends on the host immune response and metastasis usually occurs in suboptimal physiological conditions of the dog, such as immunosuppression and malnutrition [18]. In our study all the dogs with TVT metastasis had a thin to emaciated body condition with concurrent infection with Dirofilaria immitis and/or Spirocerca lupi. These conditions likely compromised the immunity of these dogs.

\section{Conclusion}

Our findings indicate that canine TVT is common in Grenada and has a relatively high metastatic rate. Therefore, it is important to emphasize the need to consider TVT as one of the differential diagnosis for the masses in extragenital locations in dogs from regions where TVT is prevalent.

\section{REFERENCES}

[1] F. Albanese, A. Poli, F. Millanta and F. Abramo, "Primary Cutaneous Extragenital Canine Transmissible Venereal Tumour with Leishmania-Laden Neoplastic Cells: A Further Suggestion of Histiocytic Origin?" Veterinary Dermatology, Vol. 13, No. 5, 2002, pp. 243-246.

[2] R. A. Foster, "Female and Male Reproductive Systems," In: M. D. McGavin and J. F. James Zachary, Eds., Pathologic Basis of Veterinary Disease, 4th Edition, Mosby, St. Louis, 2007, pp. 1306,1346.

[3] A. Gurel, B. Kuscu, E. G. Gulanber and S. S. Arun, "Transmissible Venereal Tumors Detected in the Extragenital Organs of Dogs," Israel Journal of Veterinary Medicine, Vol. 57, No. 2, 2002, pp. 1-8.

[4] K. S. Rogers, M. A. Walker and H. B. Dillon, "Transmissible Venereal Tumor: A Retrospective Study of 29 Cases," Journal of the American Animal Hospital Association, Vol. 34, 1998, pp. 463-470.

[5] A. Bastan, B. Duygu and C. Mehmet, "Uterine and Ovarian Metastasis of Transmissible Venereal Tumor in a Bitch," Turkish Journal of Veterinary and Animal Sciences, Vol. 32, No. 1, 2008, pp. 65-66.

[6] L. N. Chiti, K. Too and P. D. Sayer, "Anal Transmissible Tumor in a Dog," Zambian Journal of Veterinary Science, Vol. 1, No. 1, 1996, pp. 23-26.

[7] U. Das and K. Das, "Review of Canine Transmissible Venereal Sarcoma," Veterinary Research Communications, Vol. 24, No. 8, 2000, pp. 545-556. doi:10.1023/A:1006491918910

[8] M. Park, Y. Kim, M. Knag, S. Oh, D. Cho, N. Shin and D. Kim, "Disseminated Transmissible Venereal Tumor in a Dog," Journal of Veterinary Diagnostic Investigation, Vol. 18, No. 1, 2006, pp. 130-133. doi: $10.1177 / 104063870601800123$

[9] J. H. Rust, "Transmissible Lymphosarcoma in the Dog," Journal of the American Veterinary Medical Association, Vol. 114, No. 862, 1949, pp. 10-14.

[10] D. A. Higgins, "Observations on the Canine Transmissible Venereal Tumor as Seen in the Bahamas," Veterinary Record, Vol. 79, No. 3, 1966, pp. 67-71. doi:10.1136/vr.79.3.67

[11] O. O. Oduye, B. O. Ikede, G. O. Esuruoso and J. U. Akpokodje, "Metastatic Transmissible Venereal Tumour in Dogs," Journal of Small Animal Practice, Vol. 14, No. 10, 1973, pp. 625-637. doi:10.1111/j.1748-5827.1973.tb06399.x

[12] B. Ivoghli, "Canine Transmissible Venereal Tumor in 
Iran,” Veterinary Pathology, Vol. 14, 1977, pp. 289-290. doi: $10.1177 / 030098587701400313$

[13] A. J. Ferreira, A. Jaggy, A. P. Varejao, M. L. Ferreira, J. M. Correia, J. M. Mulas, O. Almeida, P. Oliveira and J. Prada, "Brain and Ocular Metastases from a Transmissible Venereal Tumor in a Dog," Journal of Small Animal Practice, Vol. 41, No. 4, 2000, pp. 165-168. doi:10.1111/j.1748-5827.2000.tb03187.x

[14] T. O. Abuom and J. D. Mande, "Transmissible Venereal Tumor with Subcutaneous and Bone Metastasis," The Kenya Veterinarian, Vol. 30, No. 1, 2006, pp. 10-12.

[15] S. Mukaratirwa and E. Gruys, "Canine Transmissible Venereal Tumor: Cytogenetic Origin, Immunophenotype, and Immunobiology: A Review," Veterinary Quarterly, Vol. 25, No. 3, 2003, pp. 101-111. doi:10.1080/01652176.2003.9695151
[16] L. G. Papazoglou, A. F. Koutinas, A. G. Plevraki and D. Tontis, "Primary Intranasal Transmissible Venereal Tumor in the Dog: A Retrospective Study of Six Spontaneous Cases," Journal of Veterinary Medicine Series A-Physiology Pathology Clinical Medicine, Vol. 48, No. 7, 2001, pp. 391-400.

[17] K. Gupta and N. K. Sood, "Pathological and Immunohistochemical Studies on Rare Cases of Primary ExtraGenital Transmissible Venereal Tumours in the Mammary Gland," Veterinarni Medicina, Vol. 57, No. 4, 2012, pp. 198-206.

[18] P. C. Kennedy and R. B. Miller, "Female Genital System,” In: K. V. F. Jubb, P. C. Kennedy, P. N. Nigel, Eds., Pathology of Domestic Animals, 4th Edition, Academic Press, New York, 1993, pp. 451-453. 